\title{
Concordance between measured and estimated appendicular muscle mass in adult females
}

\author{
S Lekamwasam ${ }^{1}$, J Nanayakkara ${ }^{2}$ \\ (Index words: lean muscle mass, body composition, dual-energy x-ray absorptiometry)
}

\begin{abstract}
This study assessed the accuracy of a selected formula used to estimate the appendicular muscle mass (AMM) which is linked with many clinical outcomes. A group of community-dwelling adult women $(n=80)$ had their AMM measured using dual energy $x$-ray absorptiometry (DXA). The same was estimated using a formula already published \{Skeletal muscle mass $=(0.244 \times \mathrm{BW}$ in $\mathrm{kg})+$ $(7.80 \times \mathrm{Ht}$ in meters $)+(6.6 \times \mathrm{Sex})-(0.098 \times$ Age $)+$ race 3.3 (sex $=0$ for female and 1 for male, race $=-1.2$ for Asian, 1.4 for African American and 0 for White and Hispanic). The two datasets were compared for accuracy and precision. Mean AMM measured by DXA and estimated by the formula were very close (14.8 and $14.5 \mathrm{~kg}$ ) and the difference ranged from -1.2 to $3.6 \mathrm{~kg}$. Correlation between the two datasets was high $(r=0.92)$ and the Bland-Altman plot showed an acceptable measurement agreement between the two methods. Results were independent of age and BMI. The formula used in this analysis gave an accurate estimation of the absolute AMM in women included in this study.
\end{abstract}

Ceylon Medical Journal 2015; 60: 100-102

\section{Introduction}

Low lean muscle mass (MM) is connected with cachexia, muscle atrophy and sarcopenia, three conditions broadly similar but have own characteristics [1]. Cachexia is a complex metabolic syndrome associated with an underlying illness while muscle atrophy is generally a result of physical inactivity [2]. Reduced MM is an essential criterion to define sarcopenia which refers to the loss of MM and function seen in older people [2].

Reduced MM is associated with many health consequences such as functional impairment and physical disability, reduced cardiovascular fitness and increased arterial stiffness [3-5]. Most of these studies have been done among older subjects and sarcopenia is now considered a modifiable risk factor [6].
MM can be measured by many techniques including CT, MRI, dual energy x-ray absorptiometry (DXA) and bio-impedance analysis [1]. The use of MM in clinical decision making is greatly limited and apart from BMI and calf circumference which are surrogates of MM, absolute $\mathrm{MM}$ is not used either in clinical decision making or monitoring disease progression.

Lee et al. in 2000 introduced two formulae to estimate AMM in adults [7]. They are based on anthropometric measures such as weight, height and calf circumference and other variables such as sex and ethnicity. These formulae showed a high correlation and accuracy between the estimated AMM and the measured AMM by MRI [7]. While these formulae would provide clinicians and researchers an easy and practical way of estimating the absolute AMM in human subjects, they need to be validated when applied to different populations. In this study we examined the measurement accuracy of a selected formula published by Lee et al. in a group of adult women who were participants of a previous study [7].

\section{Methods}

This study included females aged between 27-87 years selected from the field study area of the Faculty of Medicine, University of Ruhuna, using a stratified random method. These women were selected by Family Health Workers using the most recent voters' register after stratifying them to different age groups. They had an initial interview during which health related information was gathered and weight and height were measured using a digital platform weigh scale (Weigh Master International; model no BW-110 H).

All participants underwent whole body DXA scanning (Discovery by Hologic Inc, Bedford, USA) adhering to the manufacturer's protocol and the whole body images were analysed using the whole body analysis software. All scans were performed and analysed by the same technician to avoid inter-operator variability. AMM was calculated by adding the lean mass content of four limbs (Measured AMM). AMM was estimated (Estimated

${ }^{1}$ Centre for Metabolic Bone diseases, Department of Medicine and ${ }^{2}$ Department of Pharmacology, Faculty of Medicine, University of Ruhuna, Galle, Sri Lanka.

Correspondence: SL, e-mail: <slekamwasam@gmail.com>. Received 20 April and revised version accepted 25 July 2015. 
AMM) by the anthropometric prediction model described below [7]. This model was selected as the information it uses can be easily obtained in any clinical setting.

Skeletal muscle mass $=(0.244 \times$ body weight in $\mathrm{Kg})$ $+(7.80 \times$ height in meters $)+(6.6 \times$ sex $)-(0.098 \times$ age $)$ + race -3.3 .

(sex=0 for female and 1 for male, race =-1.2 for Asian, 1.4 for African American and 0 for White and Hispanic)

\section{Results}

Sample consisted of 80 females, their ages ranged from 27 to 87 years (mean 58.1 years; SD 15.5). The description of the sample is given in table 1 . None of them suffered from serious acute illness, endocrine disease, malabsorption, immobility or chronic inflammatory diseases. Twenty were on treatment for hypertension, high cholesterol or coronary artery disease. None had ever smoked or taken alcohol in the past.

Mean values of measured and estimated MM were very close (14.8 and $14.5 \mathrm{~kg}$ ) and the difference of MM, measured and estimated, ranged from -1.2 to $3.6 \mathrm{kgs}$. However, a wider SD was observed for the estimated MM. The coefficient of variation (SD/mean $\times 100)$ of measured and estimated MM were $19.6 \%$ and $29.7 \%$, respectively.

Measured and estimated MM values showed a high correlation ( $\mathrm{r}=0.92, p<0.001)$ and estimated MM accounted for $85 \%$ variation $\left(\mathrm{R}^{2}=0.85\right)$ of the measured $\mathrm{MM}$. In the regression analysis, a strong liner relationship between the two set of values was observed with a gradient of $0.62(p<0.001)$ and an intercept of $5.8(p<0.001)$ (Figure).

Table 1. Description of the sample $(n=80)$

\begin{tabular}{lll}
\hline Measurement & Range & Mean (SD) \\
\hline Age (years) & 27 to 87 & $58.1(15.5)$ \\
Weight $(\mathrm{kg})$ & 34.6 to 78.0 & $53.6(11.8)$ \\
Height $(\mathrm{m})$ & 1.37 to 1.62 & $1.48(0.06)$ \\
BMI (kg/m $\left.{ }^{2}\right)$ & 17.2 to 35.6 & $24.5(4.7)$ \\
Measured AMM (kg) & 7.6 to 21.8 & $14.8(2.9)$ \\
Estimated AMM (kg) & 7.1 to 22.5 & $14.5(4.3)$ \\
$*$ Difference (kg) & -1.2 to 3.6 & $1.6(1.2)$ \\
Medical conditions & Percentage & \\
Hypertension & $8 \%$ & \\
Diabetes & $6 \%$ & \\
Ischemic heart disease & $2 \%$ & \\
Smoking & $0 \%$ & \\
Current or past alcohol & & \\
consumption & $0 \%$ & \\
\hline Difference of measured & & \\
\hline
\end{tabular}

*Difference of measured and estimated AMM

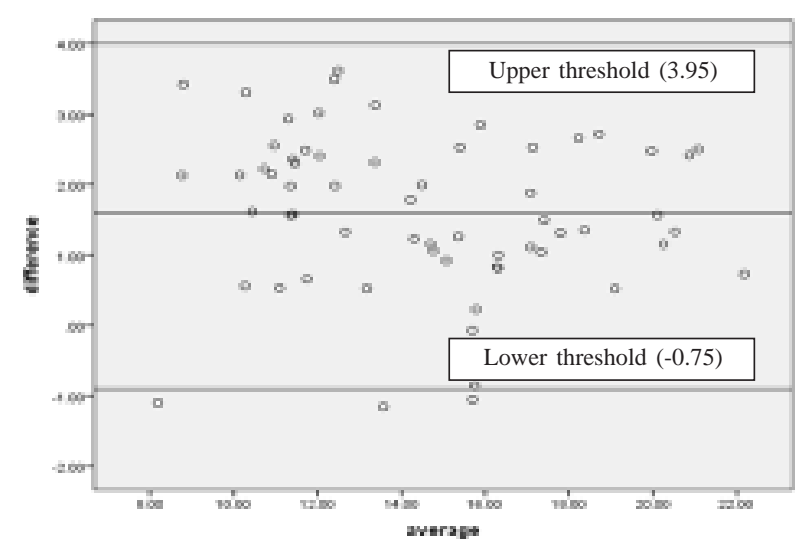

Figure. Bland-Altman plot examining the measurement agreement between the measured and estimated AMM

When the measurement agreement of the two sets of values was tested by the Bland-Altman plot, more than 95\% of values were within the limits of agreement $( \pm 1.96 \mathrm{SD}$ of the measurement difference) indicating the accuracy of the formula used to estimate the MM. When the data were analyzed based on their age and BMI, no major changes in the above results were seen. Compared to older women, young women had a stronger correlation ( $\mathrm{r}=0.92$ vs $0.84, p<0.001$ ) between the two datasets. No major difference in results was between those with BMI below 25 and those with BMI higher (table 2).

\section{Table 2. Subgroup analyses based on age and BMI}

\begin{tabular}{llll}
\hline Subgroup & $\begin{array}{l}\text { Correlation } \\
\text { coefficient(r) }\end{array}$ & $\begin{array}{l}\text { Range of } \\
\text { measurement } \\
\text { difference }(\mathrm{kg})\end{array}$ & $\begin{array}{l}\text { Mean } \\
(\mathrm{SD})(\mathrm{kg})\end{array}$ \\
\hline $\begin{array}{l}\text { Age (years) } \\
\text { Below 65 }(\mathrm{n}=45)\end{array}$ & 0.90 & -1.1 to 3.9 & $1.5(1.2)$ \\
65 and above $(\mathrm{n}=35)$ & 0.83 & -1.2 to 3.6 & $1.9(1.2)$ \\
$\begin{array}{l}\text { BMI }\left(\mathrm{kg} / \mathrm{m}^{2}\right) \\
\text { Below 25 }(\mathrm{n}=37)\end{array}$ & 0.80 & -1.1 to 3.6 & $1.7(1.2)$ \\
25 and above $(\mathrm{n}=43)$ & 0.85 & -1.1 to 3.9 & $1.6(1.1)$ \\
\hline
\end{tabular}

\section{Discussion}

This analysis showed a high accuracy of the formula used for the estimation of AMM among adult women used in this study. The absolute accuracy error ranged from -1.2 to $3.6 \mathrm{~kg}$ and it did not exceed $1 \mathrm{SD}$ of the estimated AMM (4.3 kg). The Bland-Altman plot showed more than $95 \%$ of observations lie between the limits of agreement and this further supported the accuracy of the measurement. There was a fixed error (bias) of $1.6 \mathrm{~kg}$ and this was 
significantly different from zero when tested using one-sample t-test (data not shown). However there was no proportional error to be detected in the Bland-Altman plot as the two datasets showed a uniform agreement throughout the measurement range. Further, no significant difference in results was seen among young and old or those with BMI below 25 and those above. We did not, however, include women with BMI higher than 32, hence the validity of the formula in obese women needs to be determined.

The precision error of the estimated AMM, however, was higher than that of the measured AMM by DXA technology (approximate Coefficient of variation; 30\% vs $20 \%$ ). Our data are compatible with the results of the original study which indicates that the formula can be applied to any age, gender or ethnic group [7]. Low MM is linked to functional impairment and physical disability of older Americans and Koreans [3,9]. Furthermore, among patients with established cardiovascular disease, those with low MM had lower cardiovascular fitness when compared with those with normal MM [4].

The current study had a few limitations. We studied only a group of relatively healthy adult females and the findings cannot be applied to males, other female age groups, or those with chronic diseases. We recommend this formula to estimate the AMM of adult women. Although it has an acceptable accuracy, the higher precision error observed needs to be remembered when replicate measurements are taken.

\section{References}

1. Cruz-Jentoft AJ, Baeyens JP, Bauer JM, et al. Sarcopenia: European consensus on definition and diagnosis. Report of the European Working Group on Sarcopenia in Older People. Age and Ageing 2010; 39: 412-23.

2. Evans J Willimas. Skeletal muscle loss: cachexia, sarcopenia, and inactivity. Am J Clin Nutr 2010; 91: 1123S-7S.

3. Janssen I, Heymsfield SB, Ross R. Low relative skeletal muscle mass (sarcopenia) in older persons is associated with functional impairment and physical disability. $\mathrm{J} \mathrm{Am}$ Geriatr Soc 2002; 50: 889-96.

4. Chien MY, Kuo HK, Wu YT. Sarcopenia, cardiopulmonary fitness, and physical disability in community-dwelling elderly people. Phys Ther 2010; 90: 1277-87.

5. Sampaio RA, Sewo Sampaio PY, Yamada M, et al. Arterial stiffness is associated with low skeletal muscle mass in Japanese community-dwelling older adults. Geriatr Gerontol Int 2014; 14 (Supp 1): 109-14.

6. Roubenoff R. Sarcopenia: a major modifiable cause of frailty in the elderly. J Nutr Health Aging 2000; 4: 140-2.

7. Lee RC, Wang Z, Heo M, et al. Total-body skeletal muscle mass: development and cross-validation of anthropometric prediction models. Am J Clin Nutr 2000; 72: 796-803.

8. Bland JM, Altman DG. Statistical methods for assessing agreement between two methods of clinical measurement. Lancet 1986; 327: 307-10.

9. Kim SH, Kim TH, Hwang HJ. The relationship of physical activity (PA) and walking with sarcopenia in Korean males aged 60 years and older using the Fourth Korean National Health and Nutrition Examination Survey (KNHANES IV2, 3), 2008-2009. Arch Gerontol Geriatr 2013; 56: 472-7. 